\title{
Calculation of the filtration in a heterogeneous porous medium
}

\author{
Yuri Galaguz ${ }^{1}$, Galina Safina, ${ }^{1, *}$ \\ ${ }^{1}$ Moscow state university of civil engineering, Yaroslavskoye shosse, 26, Moscow, Russia, 129337
}

\begin{abstract}
The influence of groundwater filtration on soil strength should be taken into account in the design and construction of bases and foundations. The mathematical model of the filtration of a monodisperse suspension in a heterogeneous porous medium is considered, which takes into account the change in porosity and permeability in the formation of a deposit. A numerical solution of the problem is obtained by finite differences method.
\end{abstract}

\section{Introduction}

In the design and construction of bases and foundations it is necessary to take into account the influence of groundwater filtration on soil strength. The filtration problem of a suspension in a porous medium describes the change of characteristics of a porous rock during retention of solid particles in the pores [1-3].

A porous medium is a solid body which containing thin hollow channels of various lengths and cross sections (pores). The pores connect mixing chambers where the particles mix and then pass through other pores. When a flow of suspension (carrier fluid with suspended particles) passes through a porous medium, some particles get stuck in the pores and form a deposit. The geometric model of particle retention assumes that the particles get stuck at the entrance of small pores, and freely pass through the pores of a large cross section. The scheme of particle transport and retention is given in Fig. 1.

\footnotetext{
* Corresponding author: minkinag@mail.ru
} 


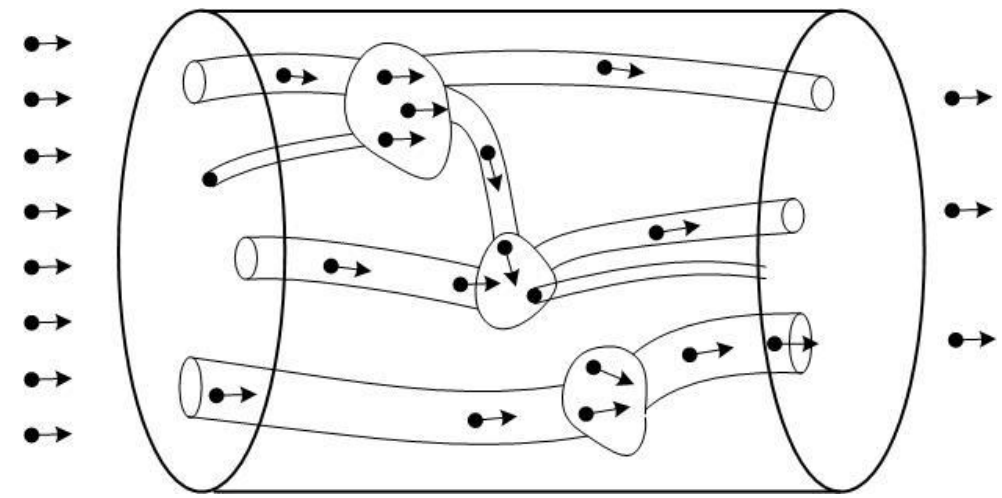

Fig. 1. Scheme of transport and retention of particles in a porous medium.

The basic model of filtration of a monodisperse suspension assumes that the velocity of particles in a porous medium is constant, and the deposit concentration does not affect the porosity and permeability of a porous medium [4-6]. More complex filtration models in a homogeneous porous medium take into account the change in porosity and permeability during the deposit formation and the inconstancy of the suspended particles velocity [7].

In a number of filtration models an exact solution is obtained [7-9], in others an asymptotic is constructed [10-14]. If analytical solution is absent, numerical methods are used [15-17].

In this paper the mathematical model of filtration of a monodisperse suspension in a heterogeneous porous medium is considered, taking into account the change in porosity and permeability during the deposit formation. The suspension of constant concentration is injected into a porous medium that does not contain suspended and retained particles. In the system of filtration equations, the unknowns are the concentrations of suspended $C(x, t)$ and retained $S(x, t)$ particles in a porous medium. A numerical solution of the problem is obtained by finite differences method [18].

\section{Formulation of the problem}

One-dimensional filtration model of suspension in a heterogeneous porous medium with changing porosity and permeability consists of two partial differential equations

$$
\begin{gathered}
\frac{\partial(g(x, S) C)}{\partial t}+\frac{\partial(f(x, S) C)}{\partial x}+\frac{\partial S}{\partial t}=0 ; \\
\frac{\partial S}{\partial t}=\Lambda(x, S) C .
\end{gathered}
$$

Here the filtration coefficient $\Lambda(x, S)$, the porosity $g(x, S)$ and permeability $f(x, S)$ are continuous functions, $\Lambda(x, S)$ is non-negative, $g(x, S)$ and $f(x, S)$ are strictly positive at $0 \leq x \leq 1, S \geq 0$.

The system of equations (1), (2) are considered in the domain

$$
\Omega=\{(x, t): 0<x<1, t>0\} .
$$

The boundary conditions for the system (1), (2) are given at the filter inlet $x=0$ and at the initial time $t=0$ : 


$$
\begin{aligned}
& \left.C(x, t)\right|_{x=0}=1, \\
& \left.C(x, t)\right|_{t=0}=0 ;\left.\quad S(x, t)\right|_{t=0}=0 .
\end{aligned}
$$

The conditions (3), (4) determine a unique solution of the problem in the domain $\Omega$.

The mobile boundary of the domain filled with particles and the empty part of a porous medium is called the concentration front of the suspended and retained particles. The concentration front is the characteristic line of the equation (1) emerging from the origin.

Since the conditions (3) and (4) do not match at the origin, according to the theory of characteristics the solution $C$ has a strong discontinuity on the concentration front; and the solution $S$ has a weak discontinuity (discontinuity of first-order derivatives) on the concentration front. Behind the concentration front in the domain $\{(x, t): 0<x<1, t>x\}$ the solution is positive $C>0, S>0$; before the front $\{(x, t): 0<x<1,0<t<x\}$ the problem (1)-(4) has a zero solution $C=0, S=0$.

The concentration front of suspended and retained particles propagates in a porous medium with variable velocity

$$
v=\frac{f(x, 0)}{g(x, 0)}
$$

\section{The exact solution at the filter inlet}

The equation (2) at filter inlet $x=0$ has the form

$$
\frac{\partial S}{\partial t}=\Lambda(0, S)
$$

Division of both sides of the equation (6) by $\Lambda(0, S)$

$$
\frac{\partial S / \partial t}{\Lambda(0, S)}=1
$$

and integration of (7) with respect to the variable $t$ gives

$$
\int_{0}^{t} \frac{\partial S / \partial t}{\Lambda(0, S)} d t=t
$$

Using the condition (4), we transform the integral on the left-hand side of (8)

$$
\int_{0}^{S(0, t)} \frac{d S}{\Lambda(0, S)}=t
$$

The equation (9) determines the concentration of retained particles at the filter inlet.

Consider the most important examples of filtration coefficients. The filter coefficient $\Lambda(x, S)$ is called blocking, if it is positive at $0 \leq x \leq 1,0 \leq S<S_{M}(x)$, and is zero when $S \geq S_{M}(x)$. In this case, the retained particles concentration is limited by the value $S_{M}(x)$.

A) For a linear blocking filtration coefficient 


$$
\Lambda(x, S)=\lambda(x)\left(S_{M}(x)-S\right)
$$

where $\lambda(x)>0, \quad S_{M}(x)>0$, the integral on the left-hand side of (9) is computed explicitly:

$$
\int_{0}^{S(0, t)} \frac{d S}{\lambda(0)\left(S_{M}(0)-S\right)}=\frac{1}{\lambda(0)} \ln \left|\frac{S_{M}(0)}{S_{M}(0)-S(0, t)}\right| .
$$

The concentration of retained particles at the filter inlet for the filtration coefficient (10) has the form

$$
S_{1}(t)=S_{M}(0)\left(1-e^{-\lambda(0) t}\right)
$$

B) For the quadratic blocking filtration coefficient

$$
\Lambda(x, S)=\lambda(x)\left(S_{M}(x)-S\right)^{2}
$$

the integral (9) is equal to

$$
\int_{0}^{S(0, t)} \frac{d S}{\lambda(0)\left(S_{M}(0)-S\right)^{2}}=\frac{S}{\lambda(0) S_{M}(0)\left(S_{M}(0)-S(0, t)\right)} .
$$

The dependence of the concentration of deposited particles on time at the filter inlet is given by formula

$$
S_{2}(t)=\frac{S_{M}^{2}(0) \lambda(0) t}{1+S_{M}(0) \lambda(0) t}
$$

Formulas (12), (14) show that the function $S(0, t)$ increases monotonically and tends to the limiting value $S_{M}(0)$ for large values of time $t$.

In Fig. 2 a), b) the graphs of the retained particles concentration at the filter inlet for the blocking filtration coefficients (12), (14) with the parameters $\lambda(0)=1, S_{M}(0)=1$ are shown.

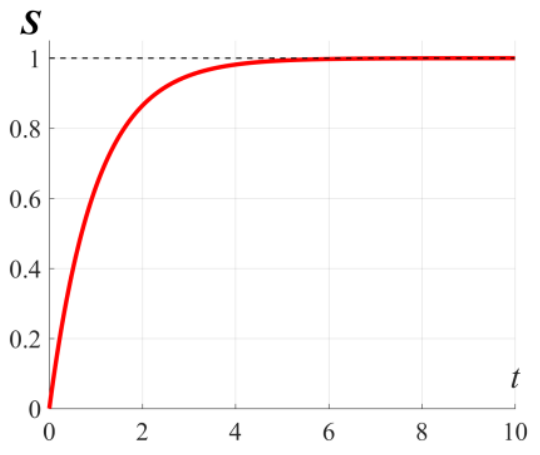

Fig. 2 a) Retained particles concentration $S_{1}(t)$.

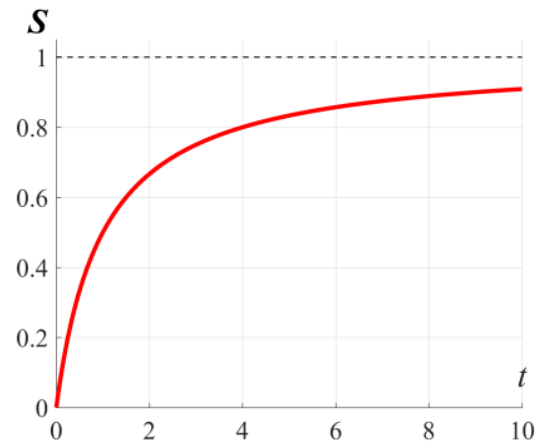

b) Retained particles concentration $S_{2}(t)$. 


\section{Numerical calculation}

The calculation is carried out by the finite difference method. For equation (1), the TVDversion of the Lax-Wendroff scheme is applied. For equation (2) the second-order RungeKutta method is used. The solution of system (1)-(4) is obtained in the domain $\{(x, t): 0<x<1,0<t<10\}$. Integration step in $x: h=0.001$, step in $t: \tau=0.0005$. The scheme satisfies the Courant-Friedrichs-Lewy condition

$$
\tau \leq \min _{x \in[0,1], t \in[0,10]}\left(\frac{g(S(x, t))}{f(S(x, t))}\right) \cdot h .
$$

Numerical calculation of the problem is carried out for coefficients of equations (1), (2)

$$
g(x, S)=(1-0,1 x)(1-0,3 S), f(x, S)=(1+0,1 x)(1-0,5 S), \Lambda(x, S)=(1+0,2 x)(1-S) .
$$

At Fig. 3 a), b) 3-D graphs of suspended and retained particles concentrations are shown.

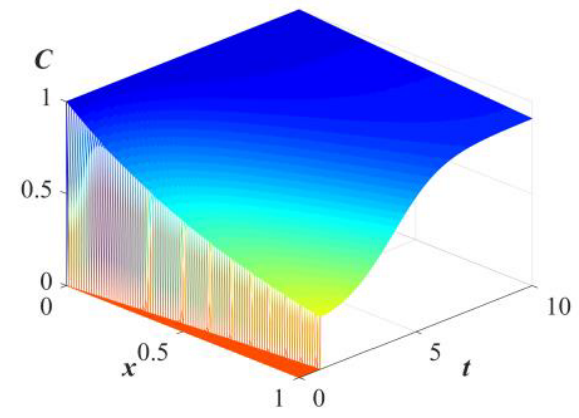

Fig. 3. a) Suspended concentration $C(x, t)$.

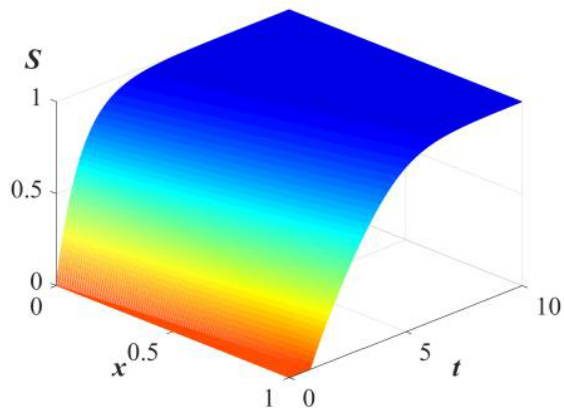

b) Retained concentration $S(x, t)$.

Graphs of suspended particles concentration at fixed time $t=0.5$ and at fixed distance $x=0.5$ are shown in Fig. 4 a), b).

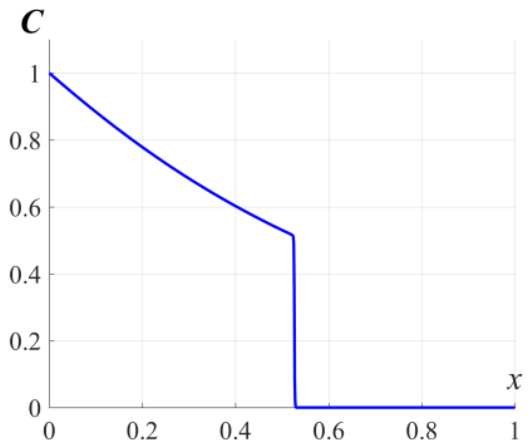

Fig. 4. a) Suspended concentration $\left.C(x, t)\right|_{t=0.5}$.

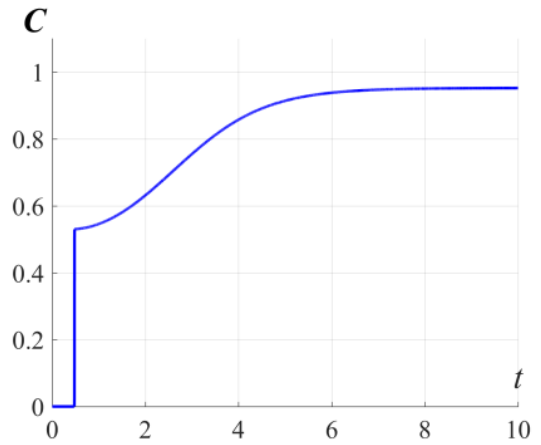

b) Suspended concentration $\left.C(x, t)\right|_{x=0.5}$.

Graphs of retained particles concentration at fixed time $t=0.5$ and at fixed distance $x=0.5$ are shown in Fig. 5 a), b). 


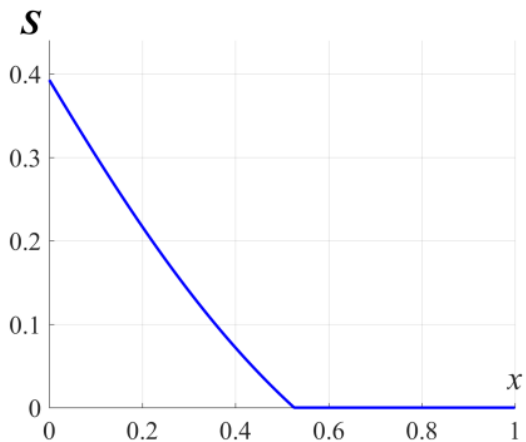

Fig. 5. a) Retained concentration $\left.S(x, t)\right|_{t=0.5}$.

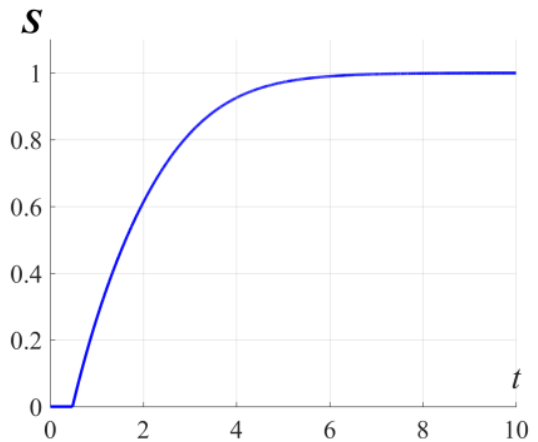

b) Retained concentration $\left.S(x, t)\right|_{x=0.5}$.

\section{Conclusion}

A numerical solution of the one-dimensional filtration problem for a monodisperse suspension in a heterogeneous porous medium is obtained. Unlike standard models that consider a homogeneous porous medium, in this paper the porosity, the permeability and the filtration coefficient depend not only on the concentration of retained particles $S(x, t)$, but also on the distance $x$ up to the filter inlet. Fig. 3 shows how the suspended and retained particles gradually fill the porous medium, moving from the inlet $x=0$ to the outlet $x=1$. The front of concentrations of suspended and retained particles is the boundary between two parts of a porous medium - empty and filled with particles. It moves with a velocity $v=1$ and reaches a filter outlet at time $t=1$. The larger the value of $x$, the later the concentration front reaches this point and retention begins (Fig. 3). With increasing deposit, the growth rate of retained particles decreases. With increasing time, the concentration of retained particles tends to the limiting value $S_{M}=1$. Since accumulation of deposit decreases for long times, the concentration of suspended particles tends to the maximum value.

\section{References}

1 K. Khilar, S. Fogler, Migration of fines in porous media (Kluwer, 1998)

2 A. Santos, P. Bedrikovetsky, S. Fontoura, J. Memb. Sci. 308 (2008)

3 F. Civan, Reservoir formation damage: fundamentals, modeling, assessment, and mitigation, (Gulf Publ. Com., 2007)

4 J. P. Herzig, D. M. Leclerc, P. Legoff, Ind. Eng. Chem. 62 (1970)

5 E.A. Vyazmina, P.G. Bedrikovetskii, A.D. Polyanin, Theor. Found. Chem. Eng. 41, 5 (2007)

6 L.I. Kuzmina, Yu.V. Osipov, Int. J. Comp. Civ. Str. Eng. 10, 32014

7 Z. You, P. Bedrikovetsky, L. Kuzmina, Abstr. Appl. Anal. 2013, ID 680693 (2013)

8 L.I. Kuzmina, Yu.V. Osipov, Struc. Mech. Anal. Const. 12017 [in Russian].

9 L.I. Kuzmina, Yu.V. Osipov, Matec Web Conf. 86, 01005 (2016)

10 Z. You, Y. Osipov, P. Bedrikovetsky, L. Kuzmina, Chem. Eng. J. 258 (2014)

11 L.I. Kuzmina, Yu.V. Osipov, Procedia Eng. 111 (2015)

12 L.I. Kuzmina, Yu.V. Osipov, Procedia Eng. 153 (2016)

13 L.I. Kuzmina, Yu.V. Osipov, Int. J. Comp. Civ. Str. Eng. 12, 12016 
14 L.I. Kuzmina, Yu.V. Osipov, Vestnik MGSU, 1 (2015)

15 V.I. Golubev, D.N. Mikhailov, Proc. MIPT 3, 2 (2011) [in Russian].

16 Yu.P. Galaguz, G.L. Safina, Procedia Eng. 153 (2016)

17 Y. Galaguz, G. Safina, Matec Web Conf. 86, 03003 (2016)

18 E.F. Toro, Riemann solvers and numerical methods for fluid dynamics, (Springer, Dordrecht, 2009) 